\title{
Unbounded perturbations of two-dimensional diffusion processes with nonlocal boundary conditions
}

\author{
Pavel Gurevich 1
}

\begin{abstract}
The existence of Feller semigroups arising in the theory of multidimensional diffusion processes is studied. Unbounded perturbations of elliptic operators (in particular, integrodifferential operators) are considered in plane bounded regions. Their domain of definition is given by a nonlocal boundary condition involving the integral over the closure of the region with respect to a nonnegative Borel measure. The support of the measure may intersect with the boundary, and the measure need not be small. We formulate sufficient conditions on unbounded perturbations of elliptic operator and on the Borel measure in the nonlocal boundary condition which guarantee that the corresponding nonlocal operator is a generator of a Feller semigroup.
\end{abstract}

1. A general form of a generator of a strongly continuous contractive nonnegative semigroup (Feller semigroup) of operators acting between the spaces of continuous functions on an interval was investigated in [1]. In the multidimensional case, it was proved that the generator of a Feller semigroup is an elliptic differential operator (possibly with degeneration) whose domain of definition consists of continuous functions satisfying nonlocal conditions which involve an integral over the closure of the region with respect to a nonnegative Borel measure [2]. The inverse problem remains open: given an elliptic integro-differential operator whose domain of definition is described by nonlocal boundary conditions, whether or not the closure of this operator is a generator of a Feller semigroup. In [3 7], the authors considered the transversal case, where the order of nonlocal terms is less than the order of local terms. The nontransversal case, where these orders coincide, was studied in [7,8] (see also the bibliography in [7]).

It was assumed in [7] that the coefficients at the nonlocal terms are less than one. In this paper, we consider nontransversal nonlocal conditions on the boundary of a plane domain and investigate the "limit case," where the coefficients at nonlocal terms may equal one at some points (the Borel measure is assumed to be atomic at these points). We formulate sufficient conditions on unbounded perturbations of elliptic operator and on the Borel measure in nonlocal conditions which guarantee that the corresponding nonlocal operator is a generator of a Feller semigroup.

Let $G \subset \mathbb{R}^{2}$ be a bounded domain with boundary $\partial G$. Consider a set $\mathcal{K} \subset \partial G$ consisting of finitely many points. Let $\partial G \backslash \mathcal{K}=\bigcup_{i=1}^{N} \Gamma_{i}$, where $\Gamma_{i}$ are open (in the topology of $\partial G) C^{\infty}$ curves. Assume that the domain $G$ is a plane angle in some neighborhood of each point $g \in \mathcal{K}$.

For any closed sets $Q \subset \bar{G}$ and $K \subset \bar{G}$ such that $Q \cap K \neq \varnothing$, we introduce the space $C_{K}(Q)=\{u \in C(Q): u(y)=0, y \in Q \cap K\}$ with the maximum-norm. Introduce the space $H_{a}^{k}(G)$ as a completion of the set of infinitely differentiable functions vanishing near $\mathcal{K}$ with respect to the norm

$$
\|u\|_{H_{a}^{k}(G)}=\left(\sum_{|\alpha| \leq k} \int_{G} \rho^{2(a+|\alpha|-k)}\left|D^{\alpha} u(y)\right|^{2} d y\right)^{1 / 2},
$$

where $a \in \mathbb{R}, k \geq 0$ is an integer, and $\rho=\rho(y)=\operatorname{dist}(y, \mathcal{K})$. For an integer $k \geq 1$, denote by $H_{a}^{k-1 / 2}(\Gamma)$ the space of traces of the functions from $H_{a}^{k}(G)$ on a smooth curve $\Gamma \subset \bar{G}$.

\footnotetext{
${ }^{1}$ This research was supported by Russian Foundation for Basic Research (project No. 07-01-00268) and the Alexander von Humboldt Foundation.
} 
We also introduce the norms in weighted spaces depending on the parameter $q>0$. Set $\|v\|_{H_{a}^{0}\left(\Gamma_{i}\right)}=\left(\int_{\Gamma_{i}} \rho^{2 a}|v(y)|^{2} d \Gamma\right)^{2}$,

$$
\begin{gathered}
\|u\|_{H_{a}^{k}(G)}=\left(\|u\|_{H_{a}^{k}(G)}^{2}+q^{k}\|u\|_{H_{a}^{0}(G)}^{2}\right)^{1 / 2}, \quad k \geq 0, \\
\|v\|_{H_{a}^{k-1 / 2}\left(\Gamma_{i}\right)}=\left(\|v\|_{H_{a}^{k-1 / 2}\left(\Gamma_{i}\right)}^{2}+q^{k-1 / 2}\|v\|_{H_{a}^{0}\left(\Gamma_{i}\right)}^{2}\right)^{1 / 2}, \quad k \geq 1 .
\end{gathered}
$$

2. Consider the differential operator $P_{0} u=\sum_{i, j=1}^{2} p_{i j}(y) u_{y_{i} y_{j}}(y)+\sum_{i=1}^{2} p_{i}(y) u_{y_{i}}(y)+$ $p_{0}(y) u(y)$, where $p_{i j}, p_{i} \in C^{\infty}\left(\mathbb{R}^{2}\right)$ are real-valued functions, $p_{0} \geq 0$, and $p_{i j}=p_{j i}$, $i, j=1,2$. We assume that there is a constant $c>0$ such that $\sum_{i, j=1}^{2} p_{i j}(y) \xi_{i} \xi_{j} \geq c|\xi|^{2}$ for $y \in \bar{G}$ and $\xi=\left(\xi_{1}, \xi_{2}\right) \in \mathbb{R}^{2}$.

Introduce operators corresponding to nonlocal terms supported near the set $\mathcal{K}$. For any set $\mathcal{M}$, we denote its $\varepsilon$-neighborhood by $\mathcal{O}_{\varepsilon}(\mathcal{M})$. Let $\Omega_{i s}\left(i=1, \ldots, N ; s=1, \ldots, S_{i}\right)$ be $C^{\infty}$ diffeomorphisms taking some neighborhood $\mathcal{O}_{i}$ of the curve $\overline{\Gamma_{i} \cap \mathcal{O}_{\varepsilon}(\mathcal{K})}$ to the set $\Omega_{i s}\left(\mathcal{O}_{i}\right)$ in such a way that $\Omega_{i s}\left(\Gamma_{i} \cap \mathcal{O}_{\varepsilon}(\mathcal{K})\right) \subset G$ and $\Omega_{i s}(g) \in \mathcal{K}$ for $g \in \overline{\Gamma_{i}} \cap \mathcal{K}$. Thus, the transformations $\Omega_{i s}$ take the curves $\Gamma_{i} \cap \mathcal{O}_{\varepsilon}(\mathcal{K})$ inside the domain $G$ and the set of their end points $\overline{\Gamma_{i}} \cap \mathcal{K}$ to itself.

Let us specify the structure of the transformations $\Omega_{i s}$. Denote by $\Omega_{i s}^{+1}$ the transformation $\Omega_{i s}: \mathcal{O}_{i} \rightarrow \Omega_{i s}\left(\mathcal{O}_{i}\right)$ and by $\Omega_{i s}^{-1}: \Omega_{i s}\left(\mathcal{O}_{i}\right) \rightarrow \mathcal{O}_{i}$ the inverse transformation. The set of points $\Omega_{i_{q} s_{q}}^{ \pm 1}\left(\ldots \Omega_{i_{1} s_{1}}^{ \pm 1}(g)\right) \in \mathcal{K}\left(1 \leq s_{j} \leq S_{i_{j}}, j=1, \ldots, q\right)$ is said to be an orbit of the point $g \in \mathcal{K}$. In other words, the orbit of a point $g \in \mathcal{K}$ is formed by the points (of the set $\mathcal{K}$ ) that can be obtained by consecutively applying the transformations $\Omega_{i_{j} s_{j}}^{ \pm 1}$ to the point $g$. The set $\mathcal{K}$ consists of finitely many disjoint orbits, which we denote by $\mathcal{K}_{\nu}$, $\nu=1, \ldots, N_{0}$.

Take a sufficiently small number $\varepsilon>0$ such that there exist neighborhoods $\mathcal{O}_{\varepsilon_{1}}\left(g_{j}\right)$, $\mathcal{O}_{\varepsilon_{1}}\left(g_{j}\right) \supset \mathcal{O}_{\varepsilon}\left(g_{j}\right)$, satisfying the following conditions: 1 . the domain $G$ is a plane angle in the neighborhood $\mathcal{O}_{\varepsilon_{1}}\left(g_{j}\right) ; 2 . \overline{\mathcal{O}_{\varepsilon_{1}}(g)} \cap \overline{\mathcal{O}_{\varepsilon_{1}}(h)}=\varnothing$ for any $g, h \in \mathcal{K}, g \neq h ; 3$. if $g_{j} \in \overline{\Gamma_{i}}$ and $\Omega_{i s}\left(g_{j}\right)=g_{k}$, then $\mathcal{O}_{\varepsilon}\left(g_{j}\right) \subset \mathcal{O}_{i}$ and $\Omega_{i s}\left(\mathcal{O}_{\varepsilon}\left(g_{j}\right)\right) \subset \mathcal{O}_{\varepsilon_{1}}\left(g_{k}\right)$.

For each point $g_{j} \in \bar{\Gamma}_{i} \cap \mathcal{K}_{\nu}$, we fix a linear transformation $Y_{j}: y \mapsto y^{\prime}\left(g_{j}\right)$ (the composition of the shift by the vector $-\overrightarrow{O g_{j}}$ and rotation) mapping the point $g_{j}$ to the origin in such a way that $Y_{j}\left(\mathcal{O}_{\varepsilon_{1}}\left(g_{j}\right)\right)=\mathcal{O}_{\varepsilon_{1}}(0), Y_{j}\left(G \cap \mathcal{O}_{\varepsilon_{1}}\left(g_{j}\right)\right)=K_{j} \cap \mathcal{O}_{\varepsilon_{1}}(0), Y_{j}\left(\Gamma_{i} \cap\right.$ $\left.\mathcal{O}_{\varepsilon_{1}}\left(g_{j}\right)\right)=\gamma_{j \sigma} \cap \mathcal{O}_{\varepsilon_{1}}(0)(\sigma=1$ or 2$)$, where $K_{j}$ is a plane angle of nonzero opening and $\gamma_{j \sigma}$ its sides.

Condition 1. Let $g_{j} \in \overline{\Gamma_{i}} \cap \mathcal{K}_{\nu}$ and $\Omega_{i s}\left(g_{j}\right)=g_{k} \in \mathcal{K}_{\nu}$; then the transformation $Y_{k} \circ \Omega_{i s} \circ$ $Y_{j}^{-1}: \mathcal{O}_{\varepsilon}(0) \rightarrow \mathcal{O}_{\varepsilon_{1}}(0)$ is the composition of rotation and homothety.

Introduce the nonlocal operators: $\mathbf{B}_{i} u=\sum_{s=1}^{S_{i}} b_{i s}(y) u\left(\Omega_{i s}(y)\right), y \in \Gamma_{i} \cap \mathcal{O}_{\varepsilon}(\mathcal{K})$, and $\mathbf{B}_{i} u=0, y \in \Gamma_{i} \backslash \mathcal{O}_{\varepsilon}(\mathcal{K})$, where $b_{i s} \in C^{\infty}\left(\mathbb{R}^{2}\right)$ are real-valued functions, $\operatorname{supp} b_{i s} \subset \mathcal{O}_{\varepsilon}(\mathcal{K})$.

Condition 2. 1. $b_{i s}(y) \geq 0, \quad \sum_{s=1}^{S_{i}} b_{i s}(y) \leq 1, \quad y \in \overline{\Gamma_{i}}$;

$$
\text { 2. } \sum_{s=1}^{S_{i}} b_{i s}(g)+\sum_{s=1}^{S_{j}} b_{j s}(g)<2, g \in \overline{\Gamma_{i}} \cap \overline{\Gamma_{j}} \subset \mathcal{K}, \quad \text { if } i \neq j \text { and } \overline{\Gamma_{i}} \cap \overline{\Gamma_{j}} \neq \varnothing \text {. }
$$

Theorem 1. Let Conditions 1 and 2 be fulfilled. Then there is a number $\delta_{0}>0$ such that, for $k=0,1,2, \ldots, \delta \in\left(0, \delta_{0}\right), q>q_{0}(\delta) \geq 0$, and $\psi_{i} \in C_{\mathcal{K}}\left(\overline{\Gamma_{i}}\right) \cap H_{a}^{k+3 / 2}\left(\Gamma_{i}\right), a=k+1-\delta$, the problem

$$
P_{0} u-q u=0, y \in G ;\left.\quad u\right|_{\Gamma_{i}}-\mathbf{B}_{i} u=\psi_{i}(y), y \in \Gamma_{i}, i=1, \ldots, N,
$$

has a unique solution $u \in C^{\infty}(G) \cap C_{\mathcal{K}}(\bar{G}) \cap H_{a}^{k+2}(G)$; moreover,

$$
\|u\|_{C(\bar{G})}+\|u\|_{H_{a}^{k+2}(G)} \leq c \sum_{i=1}^{N}\left(\left\|\psi_{i}\right\|_{C\left(\overline{\Gamma_{i}}\right)}+\left\|\psi_{i}\right\|_{H_{a}^{k+3 / 2}\left(\Gamma_{i}\right)}\right),
$$

where $c>0$ does not depend on $q, \psi_{i}$, or $u$. 
From now on, we fix $\delta \in\left(0, \delta_{0}\right)$, an integer $k \geq 2$, and $a=k+1-\delta$.

3. Consider a linear bounded operator $P_{1}: H_{a}^{k+2}(G) \rightarrow H_{a-1}^{k}(G)$.

Condition 3. 1. If a function $u \in H_{a}^{k+2}(G)$ achieves its positive maximum at a point $y^{0} \in G$, then $P_{1} u\left(y^{0}\right) \leq 0$.

2. If $u \in C(\bar{G}) \cap H_{a}^{k+2}(G)$, then the function $P_{1} u$ is bounded on $G$.

3. For all sufficiently small $\varrho>0$, we have $P_{1}=P_{1 \varrho}^{1}+P_{1 \varrho}^{2}$, where the operators $P_{1 \varrho}^{1}, P_{1 \varrho}^{2}$ : $H_{a}^{k+2}(G) \rightarrow H_{a-1}^{k}(G)$ are such that

(a) $\left\|P_{1 \varrho}^{1} u\right\|_{H_{a-1}^{k}(G)} \leq c(\varrho)\|u\|_{H_{a}^{k+2}(G)}, c(\varrho)>0$ does not depend on $u$ and $c(\varrho) \rightarrow 0$ as $\varrho \rightarrow 0$,

(b) the operator $P_{1 \varrho}^{2}$ is compact.

Note that $\mathrm{D}\left(P_{1}\right) \subset C^{k}(\bar{G} \backslash \mathcal{K}) \subset C^{2}(G)$ and $\mathcal{R}\left(P_{1}\right) \subset C^{k-2}(\bar{G} \backslash \mathcal{K}) \subset C(G)$. However, $\mathcal{R}\left(P_{1}\right) \not \subset C(\bar{G})$ in general.

Remark 1. The prototype of the abstract operators $P_{1}, P_{1 \varrho}^{1}$, and $P_{1 \varrho}^{2}$ are integral operators of the form (cf. [5, 7, 9])

$$
\begin{aligned}
P_{1} u(y) & =\int_{F}[u(y+z(y, \eta))-u(y)-(\nabla u(y), z(y, \eta))] m(y, \eta) \pi(d \eta), \\
P_{1 \varrho}^{1} u(y) & =\int_{Z \leq \varrho}[u(y+z(y, \eta))-u(y)-(\nabla u(y), z(y, \eta))] m(y, \eta) \pi(d \eta), \\
P_{1 \varrho}^{2} u(y) & =\int_{Z>\varrho}[u(y+z(y, \eta))-u(y)-(\nabla u(y), z(y, \eta))] m(y, \eta) \pi(d \eta),
\end{aligned}
$$

where $F$ is a space with a $\sigma$-algebra $\mathcal{F}$ and a Borel measure $\pi, y+z(y, \eta) \in \bar{G}$ and $\left|D_{y}^{\alpha} z(y, \eta)\right| \leq Z(\eta)$ for $y \in \bar{G}, \eta \in F,|\alpha| \leq k, Z(\eta)$ is a nonnegative $\pi$-measurable bounded function, and $m(y, \eta) \geq 0$ (some additional restrictions on the functions $z(y, \eta)$, $Z(\eta)$, and $m(y, \eta)$ should also be imposed).

4. In this paper, we consider the nonlocal conditions in the nontransversal case (see, e.g., [5] for the probabilistic interpretation):

$$
u(y)-\int_{\bar{G}} u(\eta) \mu_{i}(y, d \eta)=0, y \in \Gamma_{i}, i=1, \ldots, N ; \quad u(y)=0, y \in \mathcal{K},
$$

where $\mu_{i}(y, \cdot)$ is a nonnegative Borel measure on $\bar{G}$ such that $\mu_{i}(y, \bar{G}) \leq 1, y \in \Gamma_{i}$.

Introduce the measures $\delta_{i s}(y, \cdot)$ as follows: $\delta_{i s}(y, Q)=b_{i s}(y) \chi_{Q}\left(\Omega_{i s}(y)\right), y \in \Gamma_{i} \cap$ $\mathcal{O}_{\varepsilon}(\mathcal{K})$, and $\delta_{i s}(y, Q)=0, y \in \Gamma_{i} \backslash \mathcal{O}_{\varepsilon}(\mathcal{K})$, where $Q \subset \bar{G}$ is an arbitrary Borel set and $\chi_{Q}(\cdot)$ a characteristic function of the set $Q$.

We study those measures $\mu_{i}(y, \cdot)$ which can be represented in the form

$$
\mu_{i}(y, \cdot)=\sum_{s=1}^{S_{i}} \delta_{i s}(y, \cdot)+\alpha_{i}(y, \cdot)+\beta_{i}(y, \cdot), \quad y \in \Gamma_{i},
$$

where $\alpha_{i}(y, \cdot)$ and $\beta_{i}(y, \cdot)$ are nonnegative Borel measures to be specified below.

Denote $\operatorname{spt} \alpha_{i}(y, \cdot)=\bar{G} \backslash \bigcup_{V \in T}\left\{V \in T: \alpha_{i}(y, V \cap \bar{G})=0\right\}$ (T is the set of all open sets in $\left.\mathbb{R}^{2}\right)$.

Set $\mathbf{B}_{\alpha i} u(y)=\int_{\bar{G}} u(\eta) \alpha_{i}(y, d \eta), \mathbf{B}_{\beta i} u(y)=\int_{\bar{G}} u(\eta) \beta_{i}(y, d \eta), y \in \Gamma_{i}$.

We assume that the measures $\alpha_{i}(y, \cdot)$ and $\beta_{i}(y, \cdot)$ satisfy the following conditions (cf. [7]).

Condition 4. There exist numbers $\varkappa_{1}>\varkappa_{2}>0$ and $\sigma>0$ such that 
1. $\operatorname{spt} \alpha_{i}(y, \cdot) \subset \bar{G} \backslash \mathcal{O}_{\varkappa_{1}}(\mathcal{K}), y \in \Gamma_{i}$, and $\left\|\mathbf{B}_{\alpha i} u\right\|_{H_{a}^{k+3 / 2}\left(\Gamma_{i}\right)} \leq c\|u\|_{H_{a}^{k+2}\left(G \backslash \overline{\mathcal{O}_{\varkappa_{1}}(\mathcal{K})}\right)}$,

2. $\operatorname{spt} \alpha_{i}(y, \cdot) \subset \overline{G_{\sigma}}, y \in \Gamma_{i} \backslash \mathcal{O}_{\varkappa_{2}}(\mathcal{K})$, and $\left\|\mathbf{B}_{\alpha i} u\right\|_{H_{a}^{k+3 / 2}\left(\Gamma_{i} \backslash \overline{\mathcal{O}_{\varkappa_{2}}(\mathcal{K})}\right)} \leq c\|u\|_{H_{a}^{k+2}\left(G_{\sigma}\right)}$,

where $G_{\sigma}=\{y \in G: \operatorname{dist}(y, \partial G)<\sigma\}$.

Let $\mathcal{N}=\bigcup_{i=1}^{N}\left\{y \in \Gamma_{i}: \mu_{i}(y, \bar{G})=0\right\} \cup \mathcal{K}$ and $\mathcal{M}=\partial G \backslash \mathcal{N}$. Assume that $\mathcal{N}$ and $\mathcal{M}$ are Borel sets.

Condition 5. $\beta_{i}(y, \mathcal{M})<1$ for $y \in \Gamma_{i} \cap \mathcal{M}, i=1, \ldots, N$.

Condition 6. For any function $u \in C_{\mathcal{N}}(\bar{G})$, the functions $\mathbf{B}_{\alpha i} u$ and $\mathbf{B}_{\beta i} u$ can be extended to $\overline{\Gamma_{i}}$ in such a way that the extended functions (which we also denote by $\mathbf{B}_{\alpha i} u$ and $\mathbf{B}_{\beta i} u$, respectively) belong to $C_{\mathcal{N}}\left(\overline{\Gamma_{i}}\right)$.

We represent the measures $\beta_{i}(y, \cdot)$ in the form $\beta_{i}(y, \cdot)=\beta_{i}^{1}(y, \cdot)+\beta_{i}^{2}(y, \cdot)$, where $\beta_{i}^{1}(y, \cdot)$ and $\beta_{i}^{2}(y, \cdot)$ are nonnegative Borel measures. Let us specify them. Set $\mathcal{M}_{p}=\mathcal{O}_{p}(\overline{\mathcal{M}})$, $p>0$. Consider a cut-off function $\hat{\zeta}_{p} \in C^{\infty}\left(\mathbb{R}^{2}\right)$ such that $0 \leq \hat{\zeta}_{p}(y) \leq 1, \hat{\zeta}_{p}(y)=1$ for $y \in \mathcal{M}_{p / 2}$, and $\hat{\zeta}_{p}(y)=0$ for $y \notin \mathcal{M}_{p}$. Set $\tilde{\zeta}_{p}=1-\hat{\zeta}_{p}$. Introduce the operators

$$
\begin{gathered}
\hat{\mathbf{B}}_{\beta i}^{1} u(y)=\int_{\bar{G}} \hat{\zeta}_{p}(\eta) u(\eta) \beta_{i}^{1}(y, d \eta), \quad \tilde{\mathbf{B}}_{\beta i}^{1} u(y)=\int_{\bar{G}} \tilde{\zeta}_{p}(\eta) u(\eta) \beta_{i}^{1}(y, d \eta), \\
\mathbf{B}_{\beta i}^{2} u(y)=\int_{\bar{G}} u(\eta) \beta_{i}^{2}(y, d \eta) .
\end{gathered}
$$

Consider the Banach spaces with the norms depending on the parameter $q>0$ :

1. $H_{\mathcal{N}, a}^{k+2}(G)=C_{\mathcal{N}}(\bar{G}) \cap H_{a}^{k+2}(G),\|u\|_{H_{\mathcal{N}, a}^{k+2}(G)}=\|u\|_{C_{\mathcal{N}}(\bar{G})}+\|u\|_{H_{a}^{k+2}(G)}$,

2. $H_{\mathcal{N}, a}^{k+3 / 2}\left(\Gamma_{i}\right)=C_{\mathcal{N}}\left(\overline{\Gamma_{i}}\right) \cap H_{a}^{k+3 / 2}\left(\Gamma_{i}\right),\|v\|_{H_{\mathcal{N}, a}^{k+3 / 2}\left(\Gamma_{i}\right)}=\|v\|_{C_{\mathcal{N}}\left(\overline{\Gamma_{i}}\right)}+\|v\|_{H_{a}^{k+3 / 2}\left(\Gamma_{i}\right)}$.

Condition 7. For each sufficiently small $p>0$ the following assertions are true:

1. $\left\|\hat{\mathbf{B}}_{\beta i}^{1}\right\|_{H_{\mathcal{N}, a}^{k+2}(G) \rightarrow H_{\mathcal{N}, a}^{k+3 / 2}\left(\Gamma_{i}\right)} \rightarrow 0, p \rightarrow 0$, uniformly with respect to $q$;

2. the norms $\left\|\tilde{\mathbf{B}}_{\beta i}^{1}\right\|_{H_{\mathcal{N}, a}^{k+2}(G) \rightarrow H_{\mathcal{N}, a}^{k+3 / 2}\left(\Gamma_{i}\right)}$ are bounded uniformly with respect to $q$.

Condition 8. The operators $\mathbf{B}_{\beta i}^{2}: H_{\mathcal{N}, a}^{k+2}(G) \rightarrow H_{\mathcal{N}, a}^{k+3 / 2}\left(\Gamma_{i}\right), i=1, \ldots, N$, are compact.

Introduce the space $C_{B}(\bar{G})=\{u \in C(\bar{G}): u$ satisfy nonlocal conditions (1) $\}$. We consider the unbounded operator $\mathbf{P}: \mathrm{D}(\mathbf{P}) \subset C_{B}(\bar{G}) \rightarrow C_{B}(\bar{G})$ given by

$$
\mathbf{P} u=P_{0} u+P_{1} u, \quad u \in \mathrm{D}(\mathbf{P})=\left\{u \in C_{B}(\bar{G}) \cap H_{a}^{k+2}(G): P_{0} u+P_{1} u \in C_{B}(\bar{G})\right\} .
$$

Note that $\mathrm{D}(\mathbf{P}) \subset C^{2}(G) \cap C_{B}(\bar{G})$ due to the relation $k \geq 2$ and the Sobolev embedding theorem. The proof of the following main result is based on Theorem 1 the Hille-Iosida theorem, and the maximum principle.

Theorem 2. Let Conditions 118 hold. Then the operator $\mathbf{P}$ admits the closure $\overline{\mathbf{P}}$ : $\mathrm{D}(\overline{\mathbf{P}}) \subset C_{B}(\bar{G}) \rightarrow C_{B}(\bar{G})$ and the operator $\overline{\mathbf{P}}$ is a generator of a Feller semigroup.

6. Consider an example of nonlocal conditions in which the assumptions of the paper hold. Let $\partial G=\Gamma_{1} \cup \Gamma_{2} \cup \mathcal{K}$, where $\Gamma_{1}$ and $\Gamma_{2}$ are $C^{\infty}$ curves open and connected in the topology of $\partial G, \Gamma_{1} \cap \Gamma_{2}=\varnothing$, and $\overline{\Gamma_{1}} \cap \overline{\Gamma_{2}}=\mathcal{K}$; the set $\mathcal{K}$ consists of two points $g_{1}$ and $g_{2}$. Let $\Omega_{j}, j=1, \ldots, 4$, be nondegenerate transformations of class $C^{k+2}$ defined on a neighborhood of $\overline{\Gamma_{1}}$ and satisfying the following conditions (see Fig. 1):

1. $\Omega_{1}(\mathcal{K})=\mathcal{K}, \Omega_{1}\left(\Gamma_{1} \cap \mathcal{O}_{\varepsilon}(\mathcal{K})\right) \subset G, \Omega_{1}\left(\Gamma_{1} \backslash \mathcal{O}_{\varepsilon}(\mathcal{K})\right) \subset G \cup \Gamma_{2}$, and $\Omega_{1}(y)$ is a composition of a shift of the argument, rotation, and homothety for $y \in \overline{\Gamma_{1}} \cap \mathcal{O}_{\varepsilon}(\mathcal{K})$; 


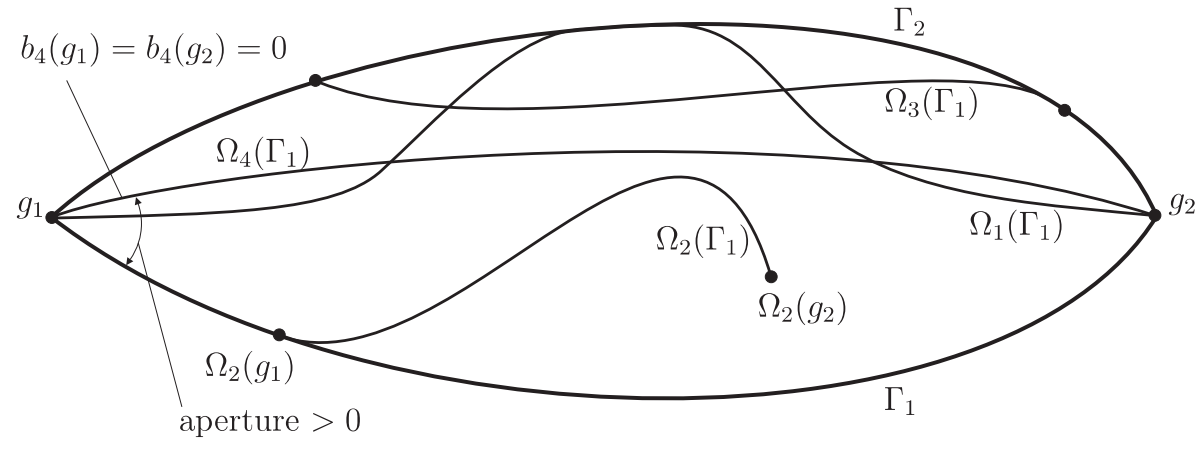

Figure 1: Nontransversal nonlocal conditions

2. there exist numbers $\varkappa_{1}>\varkappa_{2}>0$ and $\sigma>0$ such that $\Omega_{2}\left(\overline{\Gamma_{1}}\right) \subset \bar{G} \backslash \mathcal{O}_{\varkappa_{1}}(\mathcal{K})$ and $\Omega_{2}\left(\overline{\Gamma_{1}} \backslash \mathcal{O}_{\varkappa_{2}}(\mathcal{K})\right) \subset \overline{G_{\sigma}} ;$ moreover, $\Omega_{2}\left(g_{1}\right) \in \Gamma_{1}$ and $\Omega_{2}\left(g_{2}\right) \in G$

3. $\Omega_{3}\left(\overline{\Gamma_{1}}\right) \subset G \cup \Gamma_{2}$ and $\Omega_{3}(\mathcal{K}) \subset \Gamma_{2}$;

4. $\Omega_{4}\left(\overline{\Gamma_{1}}\right) \subset G \cup \overline{\Gamma_{2}}$ and $\Omega_{4}(\mathcal{K})=\mathcal{K}$; the angle between the rays tangent to $\Gamma_{1}$ and $\Omega_{4}\left(\Gamma_{1}\right)$ at the point $g_{j}$ is nonzero.

Let $b_{j} \in C^{k+2}\left(\overline{\Gamma_{1}}\right)$ and $b_{j} \geq 0, j=1, \ldots, 4$.

Consider the following nonlocal conditions:

$$
\begin{aligned}
u(y)-\sum_{j=1}^{4} b_{j}(y) u\left(\Omega_{j}(y)\right) & =0, \quad y \in \Gamma_{1}, \\
u(y) & =0, \quad y \in \overline{\Gamma_{2}} .
\end{aligned}
$$

We assume that

$$
\begin{gathered}
\sum_{j=1}^{4} b_{j}(y) \leq 1, y \in \Gamma_{1} ; \\
b_{2}\left(g_{1}\right)=0 \text { or } \sum_{j=1}^{4} b_{j}\left(\Omega_{2}\left(g_{1}\right)\right)=0 ; \quad b_{2}\left(g_{2}\right)=0 ; \quad b_{4}\left(g_{j}\right)=0 .
\end{gathered}
$$

Introduce a cut-off function $\zeta \in C^{\infty}\left(\mathbb{R}^{2}\right)$ supported in $\mathcal{O}_{\varepsilon}(\mathcal{K})$, equal to 1 on $\mathcal{O}_{\varepsilon / 2}(\mathcal{K})$, and such that $0 \leq \zeta(y) \leq 1, y \in \mathbb{R}^{2}$. Let $y \in \Gamma_{1}$, and let $Q \subset \bar{G}$ be an arbitrary Borel set; then the measures

$$
\begin{gathered}
\delta(y, Q)=\zeta(y) b_{1}(y) \chi_{Q}\left(\Omega_{1}(y)\right), \quad \alpha(y, Q)=b_{2}(y) \chi_{Q}\left(\Omega_{2}(y)\right), \\
\beta^{1}(y, Q)=(1-\zeta(y)) b_{1}(y) \chi_{Q}\left(\Omega_{1}(y)\right)+\sum_{j=3,4} b_{j}(y) \chi_{Q}\left(\Omega_{j}(y)\right), \quad \beta^{2}(y, Q)=0
\end{gathered}
$$

(for simplicity, we have omitted the subscript "1" in the notation of the measures) satisfy Conditions 1, 2, and 48.

The author is grateful to Prof. A.L. Skubachevskii for attention to this work.

\section{References}

[1] W. Feller, Trans. Amer. Math. Soc., 77, 1-30 (1954).

[2] A. D. Ventsel, Teor. Veroyatnost. i Primen., 4, 172-185 (1959); English transl.: Theory Probab. Appl., 4 (1959).

[3] K. Sato, T. Ueno, J. Math. Kyoto Univ. 4, 529-605 (1965). 
[4] J. M. Bony, P. Courrege, P. Priouret, Ann. Inst. Fourier (Grenoble) 18, (1968) 369521.

[5] K. Taira, Boundary Value Problems, Feller Semigroups, and Markov Processes, in "Interaction between Functional Analysis, Harmonic Analysis, and Probability", Vol. 40, Marcel Dekker, New York-Basel-Hong Kong, 1996.

[6] Y. Ishikawa, J. Math. Soc. Japan, 42, 171-184 (1990).

[7] E. I. Galakhov, A. L. Skubachevskii, J. Differ. Equ., 176, 315-355 (2001).

[8] A. L. Skubachevskii, Dokl. Akad. Nauk SSSR, 307, 287-291 (1989); English transl. in Soviet Math. Dokl., 40 (1990).

[9] M. G. Garroni, J. L. Menaldi, Second Order Elliptic Integro-Differential Problems, Chapman \& Hall/CRC, London-New York-Washington, D.C., 2002. 